\title{
Dual energy CT arthrography in shoulder instability: successful iodine removal with virtual non-contrast images and accurate 3D reformats of the glenoid for assessment of bone loss
}

\author{
Christoph Stern ${ }^{1,2}$ - Magda Marcon ${ }^{1,2} \cdot$ Samy Bouaicha ${ }^{2,3} \cdot$ Karl Wieser $^{2,3} \cdot$ Andrea B. Rosskopf $^{1,2} \cdot$ Reto Sutter $^{1,2}$
}

Received: 4 July 2021 / Revised: 16 September 2021 / Accepted: 19 September 2021 / Published online: 3 October 2021

(c) The Author(s) 2021, corrected publication 2022

\begin{abstract}
Objective To evaluate the image quality of dual energy CT (DECT) of the shoulder after arthrography and of virtual noncontrast (VNC) 3D reformats of the glenoid and to compare glenoid measurements on VNC 3D reformats and on 2D CTs. Materials and methods DECT arthrography $(80 \mathrm{kV} / 140 \mathrm{kV})$ was performed in 42 shoulders of 41 patients with instability using diluted iodinated contrast media $(80 \mathrm{mg} / \mathrm{ml})$. VNC images and VNC 3D reformats of the glenoid were calculated using image postprocessing. Dose parameters, CT values of intraarticular iodine and muscle, image contrast (iodine/muscle), and image quality (5-point scale: 1 = worst, $5=$ best) were evaluated. Two independent readers assessed glenoid morphology and performed glenoid measurements on $2 \mathrm{D}$ and $3 \mathrm{D}$ images.

Results Calculation of VNC images and VNC 3D reformats was successful in 42/42 shoulders (100\%). The effective dose was mean $1.95 \mathrm{mSv}( \pm 0.9 \mathrm{mSv})$. CT values of iodine and muscle were mean 1014.6 HU $( \pm 235.8 \mathrm{HU})$ and $64.5 \mathrm{HU}( \pm 8.6$ $\mathrm{HU})$, respectively, and image contrast was mean $950.2 \mathrm{HU}( \pm 235.5 \mathrm{HU})$. Quality of cross-sectional images, VNC images, and VNC 3D reformats was rated good (median 4 (4-5), 4 (3-4), 4 (3-5), respectively). Detection of an osseous defect was equal on 2D and 3D images (13/42, $P>0.99)$ with no difference for measurement of the glenoid diameter with mean $28.3 \mathrm{~mm}$ $( \pm 2.8 \mathrm{~mm})$ vs. $28.4 \mathrm{~mm}( \pm 2.9 \mathrm{~mm})(P=0.5)$, width of the glenoid defect with $3.2 \mathrm{~mm}( \pm 2.1 \mathrm{~mm})$ vs. $3.1 \mathrm{~mm}( \pm 2.3 \mathrm{~mm})$ $(P=0.84)$, surface area with $638.5 \mathrm{~mm}^{2}\left( \pm 127 \mathrm{~mm}^{2}\right)$ vs. $640.8 \mathrm{~mm}^{2}\left( \pm 129.5 \mathrm{~mm}^{2}\right)(P=0.47)$, and surface area of the defect with $46.6 \mathrm{~mm}^{2}\left( \pm 44.3 \mathrm{~mm}^{2}\right)$ vs. $47.2 \mathrm{~mm}^{2}\left( \pm 48.0 \mathrm{~mm}^{2}\right)(P=0.73)$, respectively.

Conclusion DECT shoulder arthrography is feasible and allows successful iodine removal with generation of VNC images and accurate VNC 3D reformats of the glenoid for assessment of bone loss.
\end{abstract}

Keywords Computed tomography, X-ray $\cdot$ Arthrography $\cdot$ Shoulder $\cdot$ Image enhancement

$\begin{array}{ll}\text { Abbreviations } \\ \text { CTDIvol } & \text { Volume CT dose index } \\ \text { DECT } & \text { Dual energy CT } \\ \text { DLP } & \text { Dose length product } \\ \mathrm{HU} & \text { Hounsfield unit } \\ \mathrm{kV} & \text { Kilo volt } \\ \mathrm{mAs} & \text { Milliampere seconds }\end{array}$

Christoph Stern

christoph.stern@balgrist.ch

1 Radiology, Balgrist University Hospital, Forchstrasse 340, 8008 Zurich, Switzerland

2 Faculty of Medicine, University of Zurich, Zurich, Switzerland

3 Department of Orthopaedic Surgery, Balgrist University Hospital, Forchstrasse 340, 8008 Zurich, Switzerland

$\begin{array}{ll}\text { mGy } & \text { Milligray } \\ \text { mSv } & \text { Millisievert } \\ \text { ROI } & \text { Region of interest } \\ \text { SECT } & \text { Single energy CT } \\ \text { VNC } & \text { Virtual non-contrast } \\ \text { VRT } & \text { Volume rendering technique }\end{array}$

\section{Introduction}

The 3D reformats of shoulder CT examinations generated with the volume rendering technique (VRT) are routinely used for assessment of the glenoid morphology and for quantitative measurements and are highly accurate. They are often used during preoperative planning in shoulder instability procedures, especially as the $3 \mathrm{D}$ visualization is preferred by the surgeons over the $2 \mathrm{D}$ CT reformats 
[1-7]. The 3D VRT reformats are calculated from single energy CT (SECT) data during clinical routine examinations using commercially available postprocessing software. In a subset of patients with glenoid dysplasia or previous shoulder dislocation, arthrography is performed before the CT scan to evaluate the labrum, cartilage, and the rotator cuff.

However, for SECT scans after arthrography, calculation of 3D VRT reformats is practically useless, because intraarticular iodinated contrast material overlays bone and cannot be successfully separated. In contrast to SECT, dual energy CT (DECT) allows for characterization of tissues (e.g., iodine) according to different attenuation values at different energy levels $[8,9]$. DECT has been successfully used for imaging of gout crystal depositions [10] or metal artefact reduction after metal implants [11]. However, for CT arthrography, DECT has only been tested in vitro in cow femoral condyles [12] and porcine joint cadavers [13] and was applied to shoulder patients for evaluation of labral tears [14] and for successful bone-iodine differentiation using material decomposition but without generating virtual noncontrast (VNC) images [15] or glenoid evaluation [16].

We set out to use dual energy CT after arthrography of the shoulder for clinical routine examinations. Our hypothesis was that DECT arthrography provides good image quality and allows for successful calculation of virtual non-contrast images and of accurate VNC 3D VRT reformats of the glenoid for assessment of bone loss.

Therefore, the purpose of this study was to evaluate the image quality of DECT scans of the shoulder after arthrography and of VNC 3D VRT reformats of the glenoid. Furthermore, to compare glenoid measurements on VNC 3D VRT reformats and on 2D CTs.

\section{Materials and methods}

The cantonal ethics committee approved this single center retrospective study which was conducted according to the Declaration of Helsinki.

\section{Study population}

A search of the picture archiving and communication system (PACS) of Balgrist University Hospital was performed to find patients who received a clinical dual energy CT scan of the shoulder after arthrography. Inclusion criteria were males and females aged 18 years or older with shoulder instability. Patients with an incorrect dosage of intraarticular contrast material at arthrography or with metallic screws in the glenoid after surgery were excluded.

\section{Dual energy CT technique}

All patients received a dual energy CT scan of the shoulder at Balgrist University Hospital either on a 128-slice CT scanner (SOMATOM Edge Plus, Siemens Healthineers, Erlangen, Germany; CT 1) or on a 64-slice CT scanner (SOMATOM Definition AS, Siemens Healthineers, Erlangen, Germany; CT 2) within 15 min after arthrography. The scan protocol was adapted from the protocol for the liver VNC application since no shoulder-specific manufacturer settings were available: All scans were performed in sequential technique with a $80 \mathrm{kV}$ scan followed by a second scan with $140 \mathrm{kV}$ of the same coverage in $z$-axis. Both CT scanners operated with automated tube current modulation (CARE Dose4D, reference $240 \mathrm{mAs}$ for $80 \mathrm{kV}$ and $57 \mathrm{mAs}$ for $140 \mathrm{kV}$ ), a collimation width of $0.6 \mathrm{~mm}$, a rotation time of $0.5 \mathrm{~s}$, and a pitch of 0.8 . The dose settings of the DECT scan were adjusted to the parameters of a single energy scan of the shoulder at $120 \mathrm{kV}$ (reference $150 \mathrm{mAs}$ ). The applied total dose was split automatically between the $80 \mathrm{kV}$ and the $140 \mathrm{kV}$ scan by the CT machine.

\section{Arthrography technique}

Injection of diluted iodinated contrast material into the glenohumeral joint at Balgrist University Hospital is routinely performed under conventional fluoroscopy using an anterior approach through the rotator cuff interval [17]. As there is a linear relationship of attenuation and iodine concentration for all tube voltages, the dilution of the iodinated contrast material was adapted in order to not saturate the detector of the CT machine, which occurs at $3071 \mathrm{HU}$ [12]. The total injected volume was $12 \mathrm{ml}$ for all patients. For all injections, a solution of $80 \mathrm{mg}$ iodine per milliliter was used which was achieved by injecting $1 \mathrm{ml}$ of local anesthetics followed by $11 \mathrm{ml}$ of diluted Iopamiro 200 (Iopamidol): $7 \mathrm{ml}$ Iopamiro $200+9 \mathrm{ml} \mathrm{NaCl} 0.9 \%$. With this approach, the intraarticular iodine enhancement stayed below the saturation point of the CT detector for the $80 \mathrm{kV}$ scan, which is a precondition for successful calculation of virtual non-contrast images.

\section{Image reconstruction and postprocessing}

Both the $80 \mathrm{kV}$ and $140 \mathrm{kV}$ scans were reconstructed in the axial image plane $(0.75 \mathrm{~mm})$ using a bone $(\mathrm{Br} 51)$ and a soft tissue kernel (Qr 40). Furthermore, a blended axial CT arthrogram (CT-A) dataset $(0.75 \mathrm{~mm})$ was calculated from the $80 \mathrm{kV}$ and $140 \mathrm{kV}$ scans in bone kernel 
(Br 51) using a mixing ratio of 0.3:0.7, from which axial ( $2 \mathrm{~mm})$, sagittal oblique $(2 \mathrm{~mm})$, sagittal en face $(1 \mathrm{~mm})$, and coronal oblique ( $2 \mathrm{~mm}$ ) CT arthrogram images were reconstructed.

For the calculation of 3D VRT reformats, both the axial $80 \mathrm{kV}$ and $140 \mathrm{kV}$ dataset with a $0.75-\mathrm{mm}$ section thickness in soft tissue kernel (Qr 40) were loaded into the dual energy viewer of syngo.via (VB 30, Siemens Healthineers, Erlangen, Germany). First, virtual non-contrast images with a $0.75-\mathrm{mm}$ section thickness were calculated with the shoulder VNC application which was adapted from the liver VNC application to display higher Hounsfield units. Second, 3D osseous reformats of the glenoid were generated from the VNC images using the volume rendering tool in syngo.via (Fig. 1).

\section{Image analysis}

Images were anonymized and interpreted independently by two musculoskeletal radiologists (C.S. (reader 1) with 7 and M.M. (reader 2) with 6 years of experience) on a PACS workstation. Both readers were blinded to each other and were blinded to clinical information and imaging results.

\section{Quantitative image analysis}

Scan length and CT dose parameters were extracted from the dose report: tube current-time product (mAs), volume CT dose index (CTDIvol), and dose length product (DLP). The DLP was multiplied with a standard conversion factor $k$ for the adult chest of $0.014 \mathrm{mSv} / \mathrm{mGy}^{*} \mathrm{~cm}$ to estimate the effective dose [18].

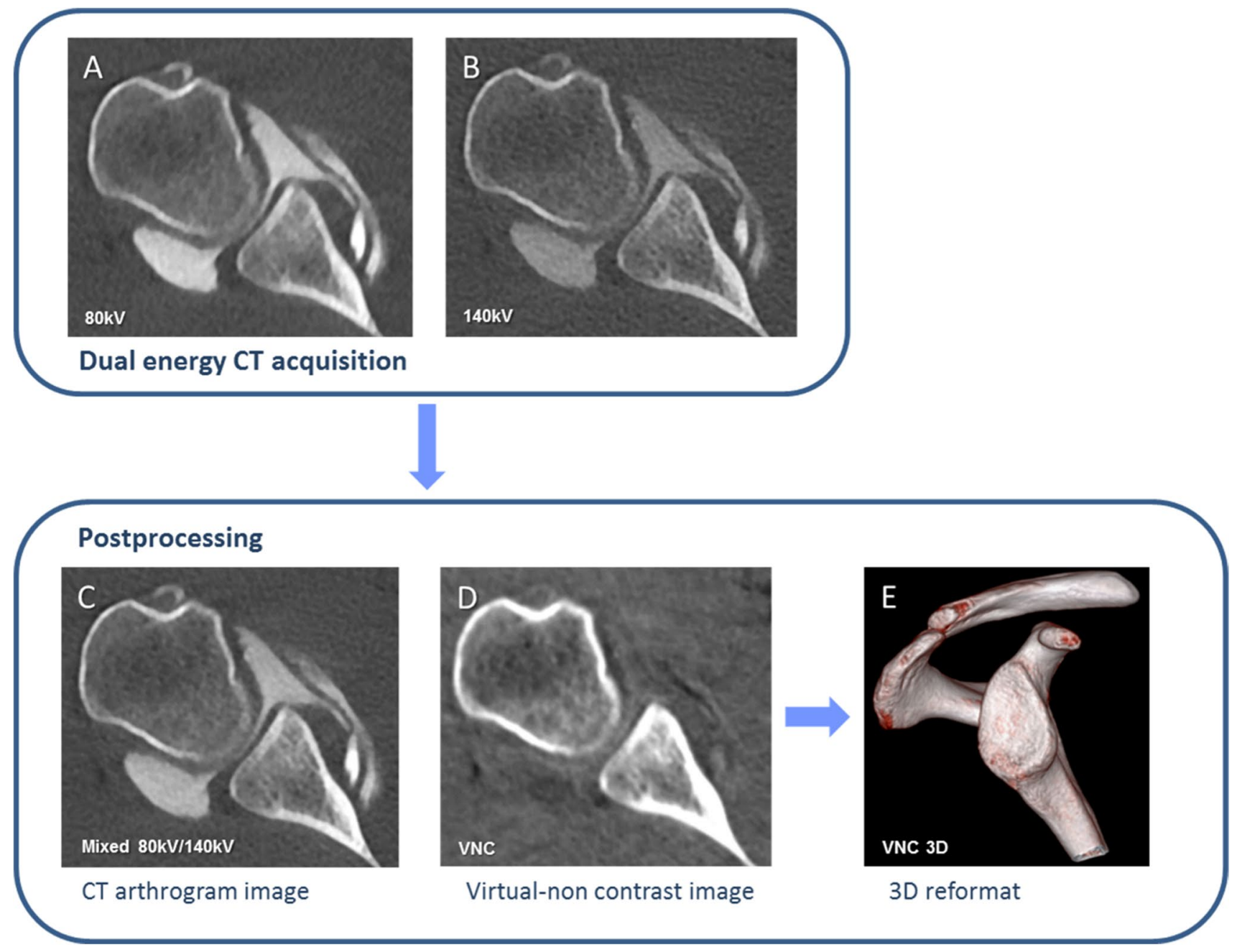

Fig. 1 Image acquisition and workflow of dual energy CT after arthrography of the shoulder. The dual energy CT scan acquires 2 datasets, one with $80 \mathrm{kV}$ tube voltage (A) and another one with $140 \mathrm{kV}$ (B). With image postprocessing, mixed CT arthrogram images $(80 \mathrm{kV} / 140 \mathrm{kV} ; \mathrm{C})$ using a mixing ratio of 0.3:0.7 and virtual non-contrast images (D) are calculated from (A) and (B). A VNC 3D osseous reformat of the glenoid (E) is calculated from (D) using the volume rendering tool 
CT values (HU) of the intraarticular iodinated contrast material were measured by reader 1 on reconstructed axial $0.75-\mathrm{mm}$ images of the $80 \mathrm{kV}$ scan in soft tissue kernel and on blended axial CT arthrogram images $(0.75 \mathrm{~mm})$ using regions of interest (ROI) of equal size $\left(20 \mathrm{~mm}^{2}\right)$. The copy and paste function of the PACS was used, allowing to position the ROIs at the identical location in both datasets. Furthermore, CT values (HU) of the deltoid muscle were also measured on the blended axial CT-A images using ROIs of the same size. Image contrast of blended CT-A images was calculated as the difference of CT values of intraarticular iodinated contrast material and deltoid muscle.

\section{Qualitative image analysis}

Reader 1 rated the following parameters on a 5-point Likert scale: overall image quality of the blended CT-A images ( $1=$ poor, $2=$ fair, $3=$ moderate, $4=$ good, $5=$ excellent $)$, image quality of virtual non-contrast images $(1=$ poor, 2 = fair, $3=$ moderate, $4=$ good, $5=$ excellent), and image quality of virtual non-contrast 3D VRT reformats of the glenoid $(1=$ poor, $2=$ fair, $3=$ moderate, $4=$ good, $5=$ excellent). Ratings are defined in Supplementary Table 1.

In a sub-analysis, the image quality was evaluated separately for the different $\mathrm{CT}$ systems.

Furthermore, CT arthrogram images of patients with surgical correlation were assessed for rotator cuff and labrum tears and for cartilage defects.

\section{Glenoid measurements}

Glenoid measurements were performed on sagittal en face 2D arthrogram images $(1 \mathrm{~mm})$ and on en face VNC 3D VRT reformats of the glenoid by both readers. The best fit-circle method was applied for all measurements, which assumes that the inferior shape of the glenoid fits a perfect circle $[2,3,19,20]$. The glenoid diameter and the glenoid surface area (Pico method) were measured on both 2D and 3D images. Both image sets were evaluated for the presence of an osseous defect. Defect size was measured as width of the glenoid defect in millimeter (mm) $[19,21]$ and as surface area of the defect in square millimeter $\left(\mathrm{mm}^{2}\right)$ for both $2 \mathrm{D}$ and 3D images [2, 3]. Reader 1 first interpreted 2D images and then 3D images, whereas reader 2 evaluated the images in the opposite order. For both readers, the interval between readouts was 2 months.

\section{Statistical analysis}

We used general descriptive statistics and reported ordinal data as median with 25 th percentile (Q1) and 75th percentile (Q3) and continuous data as mean with standard deviation (SD). To test for normal distribution, the Shapiro-Wilk test was used.

Prevalence of an osseous defect was evaluated on reconstructed 2D CT-A images and on VNC 3D VRT reformats, and the McNemar test was used for comparison. We used Bland-Altman plots [22] and the paired t-test to compare glenoid measurements on 2D and 3D images. Sub-analysis was performed to test for differences in measurements between the two CT systems. Interreader agreement was measured with intraclass correlation coefficients (ICC): ICC values $>0.75$ were interpreted as good and $>0.9$ as excellent agreement [23].

SPSS (Version 25, IBM Corporation, Armonk, NY) was used for statistical analysis with a level of significance of $<0.05$ for any value of $P$.

\section{Results}

\section{Study participants}

The PACS query revealed 52 patients with shoulder instability who received a clinical dual energy CT of the shoulder after arthrography. Six patients declined informed consent and were therefore excluded. Four patients were excluded because of metal implants and one patient because of incorrect dosage of intraarticular contrast material at arthrography. The resulting study group included 41 patients (31 male, 10 female; mean age 33.4 years \pm 13.9 years [standard deviation]) and 42 shoulders ( 1 male patient received DECT after arthrography of both shoulders).

Table 1 Scan length and CT dose parameters of patient scans

\begin{tabular}{ll}
\hline & $\begin{array}{l}\text { Dual energy CT shoulder after } \\
\text { arthrography }\end{array}$ \\
\hline Tube current & $80 \mathrm{kV}, 140 \mathrm{kV}$ \\
Tube current-time product & $80 \mathrm{kV}: 302 \mathrm{mAs}( \pm 168 \mathrm{mAs})$ \\
& $140 \mathrm{kV}: 57 \mathrm{mAs}( \pm 19 \mathrm{mAs})$ \\
CTDIvol & $11.7 \mathrm{mGy}( \pm 5.2 \mathrm{mGy})$ \\
DLP & $139.3 \mathrm{mGy} * \mathrm{~cm}( \pm 66.4 \mathrm{mGy} * \mathrm{~cm})$ \\
Scan length & $118 \mathrm{~mm}( \pm 11 \mathrm{~mm})$ \\
Effective dose $\dagger$ & $1.95 \mathrm{mSv}( \pm 0.9 \mathrm{mSv})$ \\
\hline
\end{tabular}

CT parameters were automatically adapted to patient size

${ }^{\dagger}$ Note. Effective dose (mSv) was estimated by multiplying the DLP with a standard conversion factor $\mathrm{k}$ for the adult thorax of $0.014 \mathrm{mSv} /$ mGy*cm

Values are displayed as mean with standard deviation in parentheses

Abbreviations: CTDIvol volume CT dose index, DLP dose length product, $k V$ kilo volt, $m A s$ milliampere seconds, $m G y$ milligray, $m S v$ millisievert 


\section{CT parameters, effective dose, and quantitative analysis}

CT parameters of the DECT of the shoulder after arthrography are listed in Table 1 . The estimated effective dose of the dual energy CT scans after arthrography was mean $1.95 \mathrm{mSv}$ $( \pm 0.9 \mathrm{mSv})$.

Calculation of the blended $\mathrm{CT}$ arthrogram images, of virtual non-contrast images and of VNC 3D VRT reformats of the glenoid, was successful for all shoulders $(42 / 42,100 \%)$.

$\mathrm{CT}$ values of the intraarticular iodinated contrast material were mean $1542.4 \mathrm{HU}( \pm 369 \mathrm{HU})$ for the $80 \mathrm{kV}$ scan in soft tissue kernel and 1014.6 HU $( \pm 235.8 \mathrm{HU})$ for the blended CT arthrogram images. The mean CT value of the deltoid muscle measured on CT-A images was $64.5 \mathrm{HU}( \pm 8.6 \mathrm{HU})$. The image contrast of CT-A images was mean $950.2 \mathrm{HU}$ $( \pm 235.5 \mathrm{HU})$ between intraarticular iodinated contrast material and deltoid muscle.

\section{Qualitative image analysis}

CT arthrogram images, VNC images, and VNC 3D VRT reformats were of good quality: The overall image quality of the CT-A images was median 4 (4-5), the image quality of virtual non-contrast images was median 4 (3-4), and the image quality of virtual non-contrast 3D VRT reformats of the glenoid was median 4 (3-5) (Figs. 2 and 3).

For CT 1 and CT 2, the image quality of CT arthrogram images, VNC images, and VNC 3D VRT reformats were median 4 (4-5) and 5 (4-5), 4 (3-4) and 4.5 (4-5), and 4 (3-4.25) and 5 (3.25-5), respectively. However, the number of patients scanned on CT 1 was more than 4 times higher than on CT 2 (34 vs. 8 patients).

Sub-analysis of CT arthrogram images of patients with shoulder surgery $(n=8)$ revealed labrum tears in 5 patients, rotator cuff tears in 1 patient, and cartilage defects in 3 patients, all of which were confirmed surgically.

\section{Glenoid measurements}

Glenoid measurements were successful on all sagittal en face 2D CT arthrogram images $(42 / 42,100 \%)$ and on all en face VNC 3D VRT reformats of the glenoid $(42 / 42,100 \%)$. No difference was observed for the glenoid diameter with mean $28.3 \mathrm{~mm}( \pm 2.8 \mathrm{~mm})$ measured on 2D images compared to mean $28.4 \mathrm{~mm}( \pm 2.9 \mathrm{~mm})$ measured on VNC 3D VRT reformats $(P=0.5)$. The glenoid surface area was also not different with mean $638.5 \mathrm{~mm}^{2}\left( \pm 127 \mathrm{~mm}^{2}\right)$ on $2 \mathrm{D}$ images vs. mean $640.8 \mathrm{~mm}^{2}\left( \pm 129.5 \mathrm{~mm}^{2}\right)$ on VNC 3D images $(P=0.47)$.
Fig. 2 A 20-year-old female with anterior glenohumeral instability. Dual energy CT after arthrography of the right shoulder with reformatted axial (A) and coronal oblique (B) CT arthrogram (CT-A) images clearly show the articular cartilage (black arrows), labrum (black arrow heads), and tendons of the rotator cuff (white arrows). The cortical and trabecular structure of the humeral head and of the glenoid is clearly visible. Reformatted axial (C) and coronal oblique (D) images of the virtual non-contrast (VNC) dataset show full subtraction of the intraarticular iodinated contrast material (open arrows). Note substance loss and fraying of the inferior labrum in (B) because of Bankart lesion. Image contrast between iodine and soft tissue was 1170 Hounsfield units
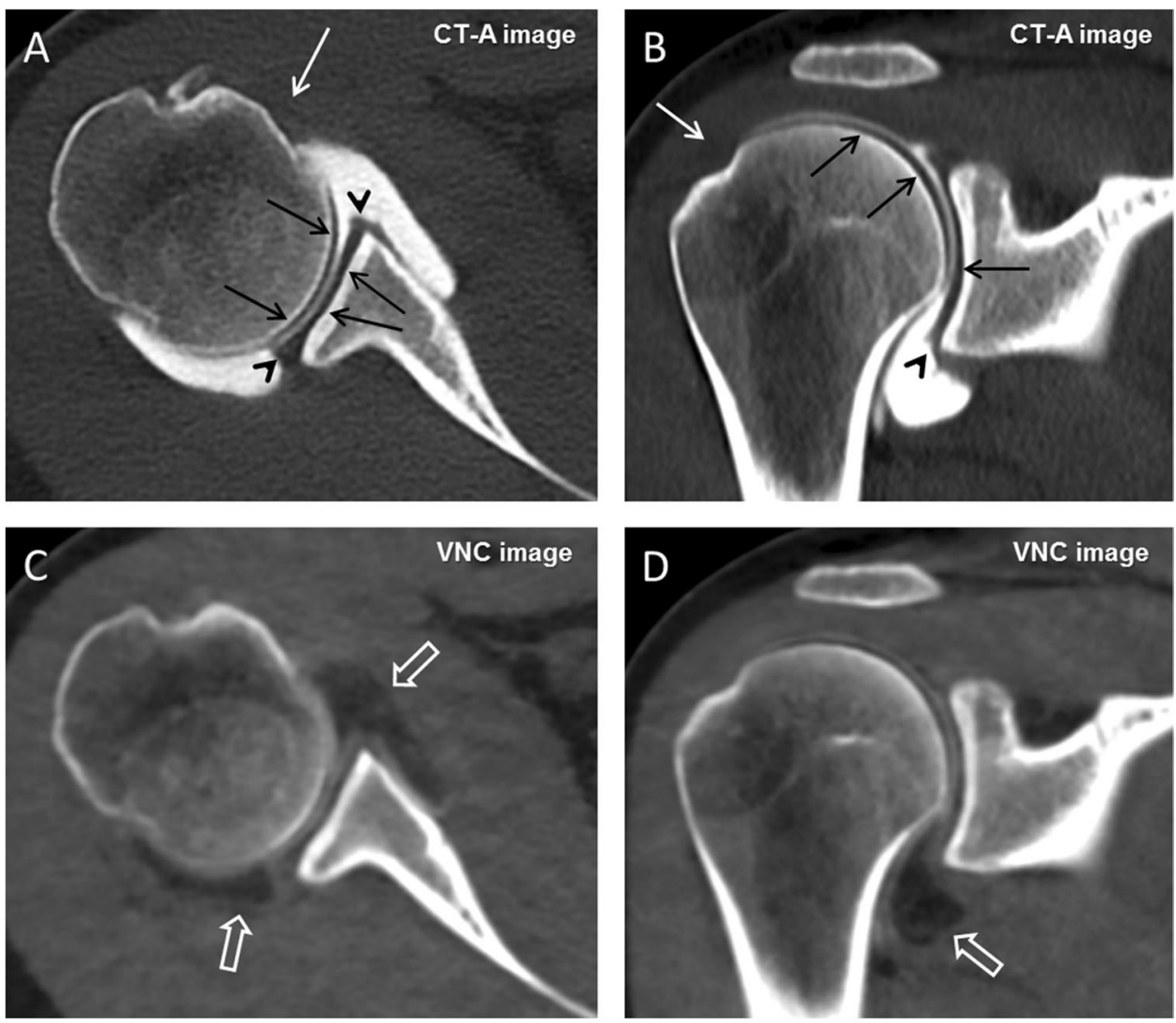

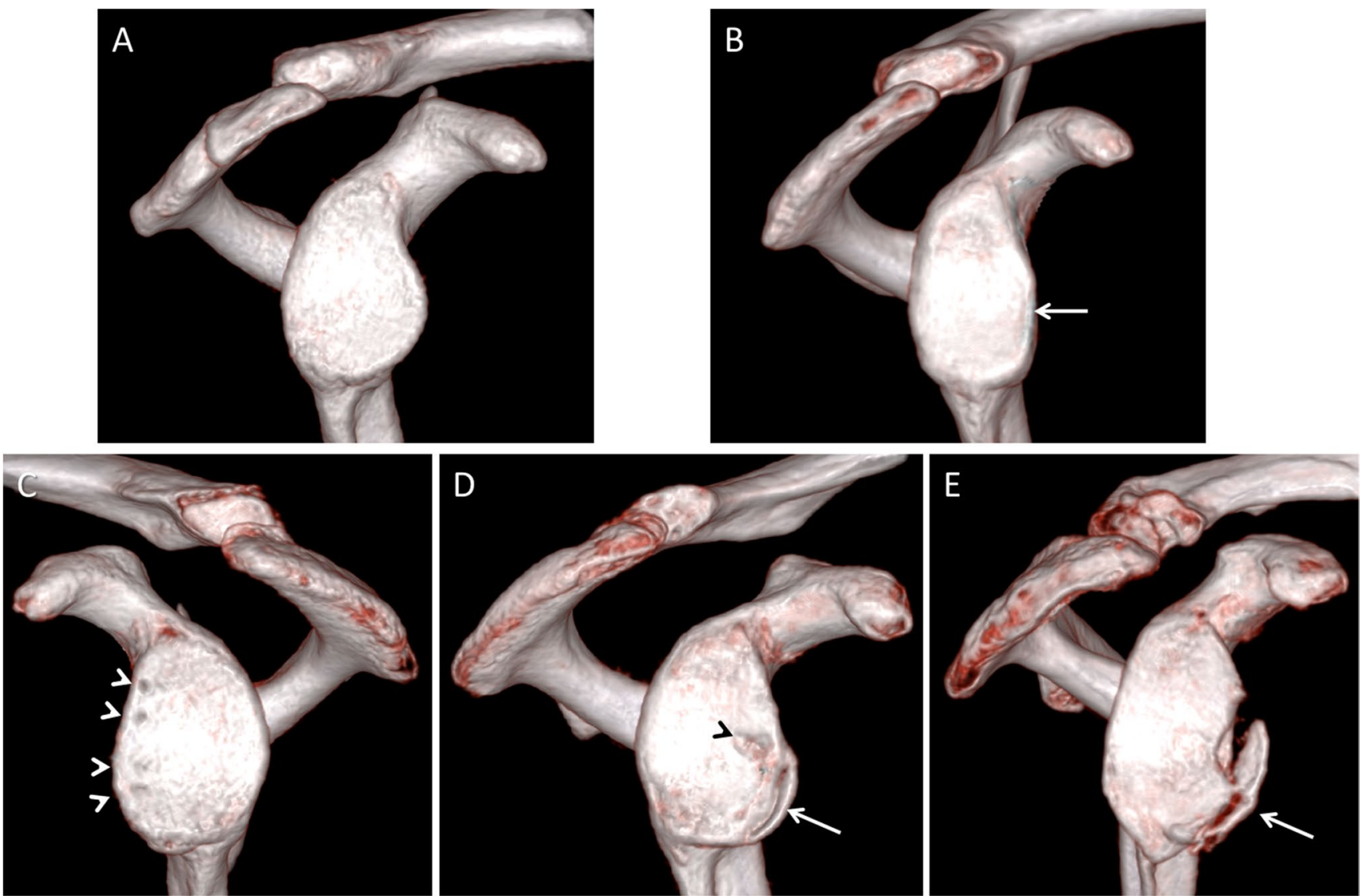

Fig. 3 Examples of VNC 3D VRT reformats of the glenoid. A A 25-year-old male with normal shaped glenoid without bone loss. B A 20-year-old female with anterior glenohumeral instability. The anteroinferior glenoid appears straight suggesting a compression fracture (arrow). C A 25-year-old male with evidence of anterior labrum

Detection of an osseous defect was equal on sagittal en face 2D CT-A images $(13 / 42,31 \%$; $95 \%$ confidence interval [CI]: $18.6 \%, 45.8 \%)$ and on en face VNC 3D VRT reformats $(13 / 42,31 \%$; 95\% CI: $18.6 \%, 45.8 \%)(P>0.99)$ with no discrepant cases. There was no difference for the width of the osseous glenoid defect with mean $3.2 \mathrm{~mm}( \pm 2.1 \mathrm{~mm})$ measured on $2 \mathrm{D}$ images compared to $3.1 \mathrm{~mm}( \pm 2.3 \mathrm{~mm})$ measured on VNC 3D images $(P=0.84)$, nor was the surface area of the osseous glenoid defect measured different with mean $46.6 \mathrm{~mm}^{2}\left( \pm 44.3 \mathrm{~mm}^{2}\right)$ and $47.2 \mathrm{~mm}^{2}( \pm 48.0$ $\left.\mathrm{mm}^{2}\right)(P=0.73)$, respectively (Fig. 4). Figure 5 shows the corresponding Bland-Altman plots. The range between the lower and upper limit was as follows: $1.8 \mathrm{~mm}$ for the glenoid diameter, $81.5 \mathrm{~mm}^{2}$ for the glenoid surface area, $1.6 \mathrm{~mm}$ for the width of the osseous glenoid defect, and $24.1 \mathrm{~mm}^{2}$ for the surface area of the osseous glenoid defect. Sub-analysis also revealed no difference for each category of glenoid measurements performed on 2D and 3D images for examinations on CT $1(n=34 ; P=0.76-0.99)$ or on CT $2(n=8 ; P=0.36-0.40)$. repair with visible drill holes (arrow heads). D A 22-year-old male with partial fibrous consolidation of a small anteroinferior osseous Bankart fragment (arrow) and a small osseous cyst (arrow head). E A 59-year-old male with recurrent shoulder instability and a large displaced osseous Bankart fragment anterior (arrow)

Agreement between readers for all glenoid measurements was excellent on 2D images (ICC 0.98) and excellent on VNC 3D VRT reformats (ICC 0.98).

\section{Discussion}

We showed that DECT shoulder arthrography is feasible for clinical routine examinations and allows successful iodine removal using material decomposition with generation of virtual non-contrast (VNC) images and accurate 3D VRT reformats of the glenoid for assessment of bone loss.

With a concentration of $80 \mathrm{mg}$ iodine per $\mathrm{ml}$ contrast solution injected into the joint we were able to acquire DECT arthrogram images in diagnostic quality and successfully calculate both cross-sectional VNC images as well as VNC 3D VRT reformats for all clinical examinations. Subhas et al. showed diagnostic image quality for CT scans of human knee cadavers performed in single energy technique with $80 \mathrm{kV}$ after arthrography using 
Fig. 4 A 28-year-old male with bony Bankart lesion of the anteroinferior glenoid. Dual energy CT after arthrography of the right shoulder with reformatted sagittal en face 2D arthrogram images (A and C) and en face 3D VNC VRT reformats of the glenoid (B and D). Measurement of the glenoid diameter (green lines) using the best fit circle method was comparable with $30.9 \mathrm{~mm}$ on the 2D image (A) and $31.4 \mathrm{~mm}$ on the 3D image (B). The width of the glenoid defect was measured identical with $3.1 \mathrm{~mm}$ (yellow lines in (A) and (B)).

The glenoid surface area (green circles) was also measured similar on the 2D image $(\mathbf{C})$ and 3D image (D) with $751 \mathrm{~mm} 2$ and $775 \mathrm{~mm} 2$, respectively. Similarly, the surface area of the osseous glenoid defect (yellow areas) was measured with 49 $\mathrm{mm} 2$ on image (C) and $44 \mathrm{~mm} 2$ on image (D)
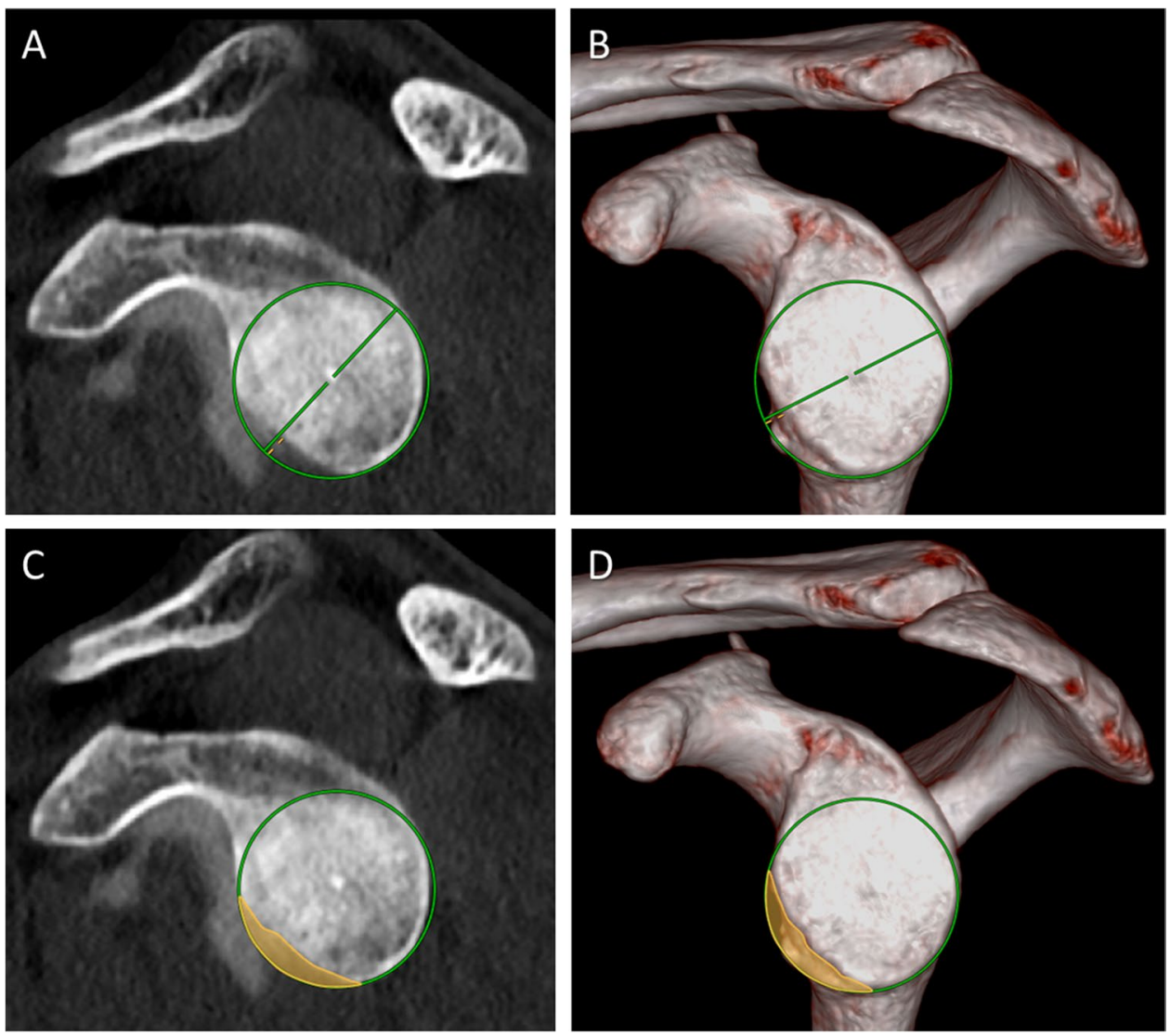

$60 \mathrm{mg}$ iodine per ml solution. Furthermore, they demonstrated that the saturation point of the CT detector was reached at $93 \mathrm{mg}$ iodine per $\mathrm{ml}$ solution for both the $80 \mathrm{kV}$ SECT and the $80 \mathrm{kV}$ scan of the $80 \mathrm{kV} / 140 \mathrm{kV}$ DECT scan in a phantom [12].

An et al. determined in a 2014 phantom study the optimal contrast dose at $60 \mathrm{mg}$ iodine/ml and the energy level at $72 \mathrm{keV}$ for DECT arthrography of the shoulder using virtual monochromatic spectral imaging. The optimized protocol was then applied to 23 patients, which resulted in $36 \%$ reduction of image noise and in $45 \%$ reduction of beam-hardening artifacts compared to the standard protocol [15]. The authors only evaluated material decomposition; however, no VNC images or 3D VNC reformats were calculated.

In our study, evaluating 42 shoulders in 41 patients, we observed excellent image contrast with a mean difference of $950 \mathrm{HU}$ between the intraarticular iodinated contrast material and the soft tissues on CT-A images, which allows to confidently assess the labrum, the rotator cuff, and the articular cartilage for pathology. In all of our patients with subsequent shoulder surgery $(n=8)$, the labrum, rotator cuff, and cartilage lesions identified on CT-A images were surgically confirmed. In their study with 47 shoulder patients, Foti et al. demonstrated that DECT arthrography is equal to magnetic resonance arthrography in detecting labral tears
[14]. Further, our results were in accordance with the results of An et al., who found an image contrast above $800 \mathrm{HU}$ between the intraarticular iodine contrast agent and soft tissues for the optimized virtual monochromatic spectral imaging DECT protocol with $60 \mathrm{mg}$ iodine/ml [15]. Therefore, we believe that $80 \mathrm{mg}$ iodine/ml solution is appropriate in DECT shoulder arthrography for both accurate assessment of the internal structures and successful iodine removal. The virtual monochromatic imaging technique, however, only decreases the attenuation of iodinated contrast material at high $\mathrm{keV}$ and is inferior to the VNC technique in generating virtual non-contrast images, which was shown by the results of Sandhu et al. [16]. We used the virtual unenhanced technique to calculate virtual non-contrast images from the $80 \mathrm{kV}$ and $140 \mathrm{kV}$ DECT dataset using the customized liver VNC application in syngo.via, which allowed for subtraction of iodinated intraarticular contrast agent.

Chai et al. also used the virtual unenhanced technique in an in vitro study with porcine joints and showed successful calculation of virtual non-contrast images and VNC $3 \mathrm{D}$ osseous reformats from DECT scans $(80 \mathrm{kV} / 140 \mathrm{kV})$ at $75 \mathrm{mg}$ iodine $/ \mathrm{ml}$. In their study, subtraction and image calculation failed for all higher contrast agent dosages, because the demonstrable upper HU limit of the CT detector was reached and consequently the subtraction 

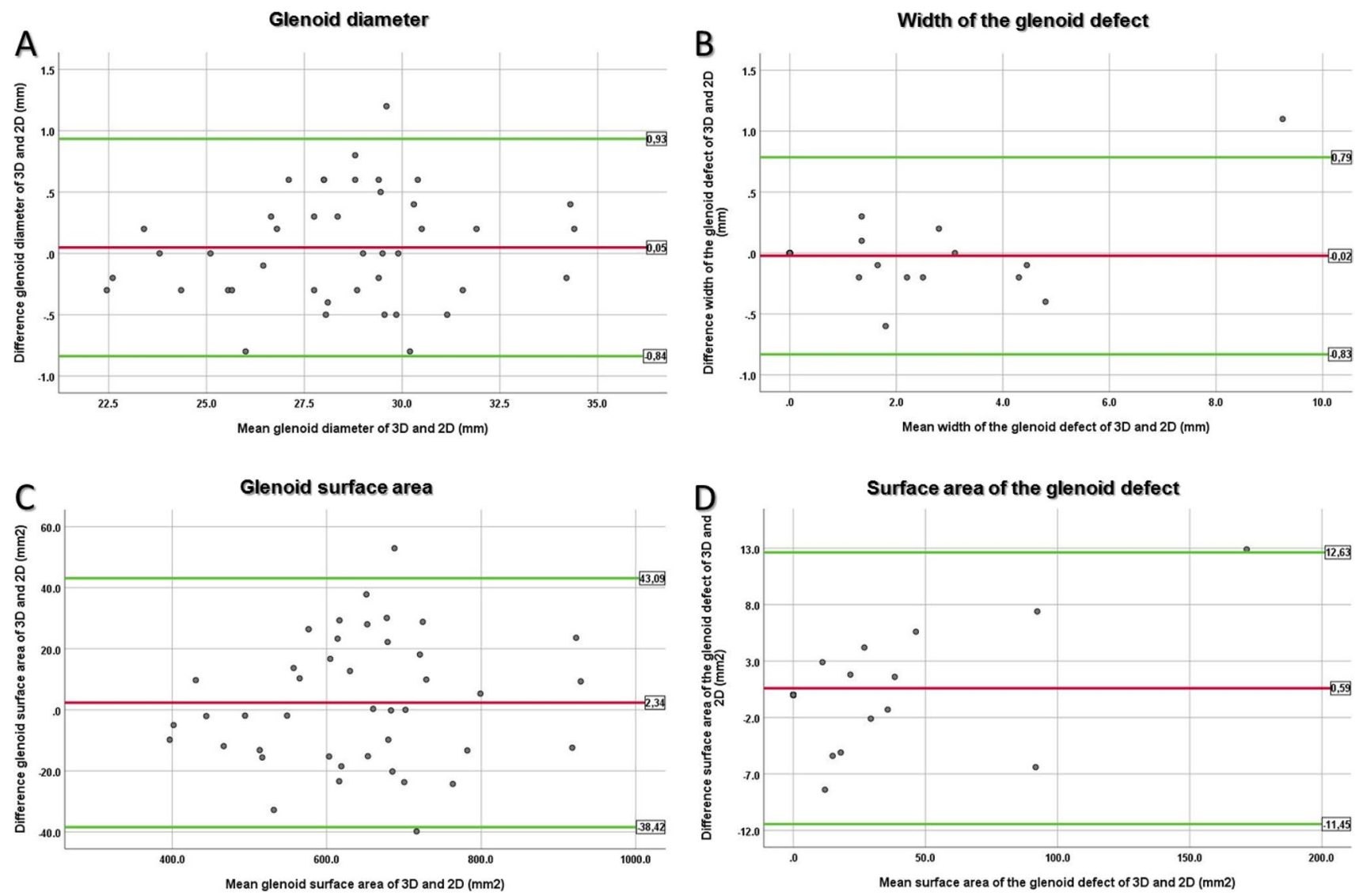

Fig. 5 Bland-Altman plots for the glenoid diameter (A), width of the glenoid defect $(\mathbf{B})$, glenoid surface area $(\mathbf{C})$, and surface area of the glenoid defect (D). The upper limit, mean value, and lower limit are

displayed in the boxes on the right of each plot. Note: Data in (A) and (B) are displayed in millimeters ( $\mathrm{mm})$ and in $(\mathbf{C})$ and $(\mathbf{D})$ in square millimeters $\left(\mathrm{mm}^{2}\right)$

algorithm of the $\mathrm{VNC}$ application was unable to recognize the material as iodine $[12,13]$. Our study results were in accordance with successful calculation of VNC images and VNC 3D VRT reformats for all study participants using $80 \mathrm{mg}$ iodine $/ \mathrm{ml}$.

Our results showed that detection of an osseous defect of the glenoid was accurate on VNC 3D VRT reformats, with no discrepant cases compared to 2D CT. In a study with 7 shoulder cadavers, Rerko et al. also demonstrated a high correlation between true anteroinferior glenoid bone loss vs. predicted bone loss on 3D CT $(r=0.875)$ and on 2D CT $(r=0.831)$, respectively, with comparable prediction errors for 3D CT and 2D CT. They recommend to use 3D CT as the most accurate, reliable, and reproducible imaging modality to estimate anteroinferior glenoid bone loss [4].

Sugaya et al. evaluated glenoid morphology in 100 shoulders with recurrent anterior glenohumeral instability and found that in shoulders with abnormal morphology discovered on 3D CT, appearance during arthroscopy was comparable [5].

Margarelli et al. discovered 97\% agreement between 2D CT and 3D VRT reformats in detecting an osseous defect of the glenoid in 100 patients with unilateral anterior glenohumeral instability. Furthermore, they showed a mean difference of $0.62 \%$ between the 2 methods for measurement of the percentage of glenoid bone loss using the Pico method [7]. Our study results were in accordance as we did not find a statistically significant difference between 2D CT and VNC 3D VRT reformats for measurement of the glenoid diameter $(P=0.5)$, the glenoid surface area $(P=0.47)$, the width of the glenoid defect $(P=0.84)$, or the surface area of the glenoid defect $(P=0.73)$. Therefore, we conclude that the subtraction algorithm of the VNC application works properly and 3D VRT reformats are accurate.

With a CTDIvol of mean $11.7 \mathrm{mGy}$ and an effective dose of mean $1.95 \mathrm{mSv}$, the dual energy CT scans of our study were below the radiation dose reported in the literature. An et al. reported a CTDIvol of $17.8 \mathrm{mGy}$ for DECT arthrography of the shoulder in 23 patients using virtual monochromatic spectral imaging [15]. Biswas et al. reported a 
CTDIvol of mean $19.5 \mathrm{mGy}$ and an effective dose of mean $2.06 \mathrm{mSv}$ for non-contrast CT scans of the shoulder with $120 \mathrm{kV}$ in 20 patients [24].

A limitation of our study was that patients were scanned on 2 different CT scanners. However, acquisition parameters were adjusted to equal dose, and the same dual energy protocols were used, which resulted in comparable image quality and no difference in glenoid measurements according to a sub-analysis for examinations performed on CT 1 and 2. Furthermore, the dual energy CT protocol incorporated a 70-s delay between the $80 \mathrm{kV}$ and the $140 \mathrm{kV}$ scan since it was derived from the liver VNC protocol. In order for the VNC application to work, deactivation of the delay was not possible. However, we did not experience significant patient motion during the two scans which could possibly negatively affect image quality.

In summary, dual energy CT arthrography of the shoulder for clinical routine examinations is feasible and showed good image quality at a dose comparable to single energy shoulder CT. With image postprocessing, diagnostic virtual noncontrast 3D VRT reformats of the glenoid were successfully calculated, which allow for 3D assessment of glenoid morphology and bone loss and accurate glenoid measurements.

Supplementary Information The online version contains supplementary material available at https://doi.org/10.1007/s00256-021-03916-3.

Funding Open access funding provided by University of Zurich.

\section{Declarations}

Conflict of interest The authors declare no competing interests.

Open Access This article is licensed under a Creative Commons Attribution 4.0 International License, which permits use, sharing, adaptation, distribution and reproduction in any medium or format, as long as you give appropriate credit to the original author(s) and the source, provide a link to the Creative Commons licence, and indicate if changes were made. The images or other third party material in this article are included in the article's Creative Commons licence, unless indicated otherwise in a credit line to the material. If material is not included in the article's Creative Commons licence and your intended use is not permitted by statutory regulation or exceeds the permitted use, you will need to obtain permission directly from the copyright holder. To view a copy of this licence, visit http://creativecommons.org/licenses/by/4.0/.

\section{References}

1. Chuang TY, Adams CR, Burkhart SS. Use of preoperative threedimensional computed tomography to quantify glenoid bone loss in shoulder instability. Arthroscopy. 2008;24(4):376-82.

2. Barchilon VS, Kotz E, Barchilon Ben-Av M, Glazer E, Nyska M. A simple method for quantitative evaluation of the missing area of the anterior glenoid in anterior instability of the glenohumeral joint. Skeletal Radiol. 2008;37(8):731-6.
3. Bois AJ, Fening SD, Polster J, Jones MH, Miniaci A. Quantifying glenoid bone loss in anterior shoulder instability: reliability and accuracy of 2-dimensional and 3-dimensional computed tomography measurement techniques. Am J Sports Med. 2012;40(11):2569-77.

4. Rerko MA, Pan X, Donaldson C, Jones GL, Bishop JY. Comparison of various imaging techniques to quantify glenoid bone loss in shoulder instability. J Shoulder Elbow Surg. 2013;22(4):528-34.

5. Sugaya H, Moriishi J, Dohi M, Kon Y, Tsuchiya A. Glenoid rim morphology in recurrent anterior glenohumeral instability. J Bone Joint Surg Am. 2003;85(5):878-84.

6. Walter WR, Samim M, LaPolla FWZ, Gyftopoulos S. Imaging quantification of glenoid bone loss in patients with glenohumeral instability: a systematic review. AJR Am J Roentgenol. 2019;212(5):1096-105.

7. Magarelli N, Milano G, Baudi P, Santagada DA, Righi P, Spina $\mathrm{V}$, et al. Comparison between 2D and 3D computed tomography evaluation of glenoid bone defect in unilateral anterior glenohumeral instability. Radiol Med. 2012;117(1):102-11.

8. Mallinson PI, Coupal TM, McLaughlin PD, Nicolaou S, Munk PL, Ouellette HA. Dual-energy CT for the musculoskeletal system. Radiology. 2016;281(3):690-707.

9. Johnson TR, Krauss B, Sedlmair M, Grasruck M, Bruder H, Morhard D, et al. Material differentiation by dual energy CT: initial experience. Eur Radiol. 2007;17(6):1510-7.

10. Gamala M, Jacobs JWG, van Laar JM. The diagnostic performance of dual energy CT for diagnosing gout: a systematic literature review and meta-analysis. Rheumatology (Oxford). 2019;58(12):2117-21.

11. Bamberg F, Dierks A, Nikolaou K, Reiser MF, Becker CR, Johnson TR. Metal artifact reduction by dual energy computed tomography using monoenergetic extrapolation. Eur Radiol. 2011;21(7):1424-9.

12. Subhas N, Freire M, Primak AN, Polster JM, Recht MP, Davros WJ, et al. CT arthrography: in vitro evaluation of single and dual energy for optimization of technique. Skeletal Radiol. 2010;39(10):1025-31.

13. Chai JW, Choi JA, Choi JY, Kim S, Hong SH, Kang HS. Visualization of joint and bone using dual-energy $\mathrm{CT}$ arthrography with contrast subtraction: in vitro feasibility study using porcine joints. Skeletal Radiol. 2014;43(5):673-8.

14. Foti G, Mantovani W, Catania M, Avanzi P, Caia S, Zorzi C, et al. Evaluation of glenoid labral tears: comparison between dualenergy CT arthrography and MR arthrography of the shoulder. Radiol Med. 2020;125(1):39-47.

15. An C, Chun YM, Kim S, Lee YH, Yun MJ, Suh JS, et al. Dualenergy computed tomography arthrography of the shoulder joint using virtual monochromatic spectral imaging: optimal dose of contrast agent and monochromatic energy level. Korean J Radiol. 2014;15(6):746-56.

16. Sandhu R, Aslan M, Obuchowski N, Primak A, Karim W, Subhas N. Dual-energy CT arthrography: a feasibility study. Skeletal Radiol. 2021;50(4):693-703.

17. Depelteau H, Bureau NJ, Cardinal E, Aubin B, Brassard P. Arthrography of the shoulder: a simple fluoroscopically guided approach for targeting the rotator cuff interval. AJR Am J Roentgenol. 2004;182(2):329-32.

18. Christner JA, Kofler JM, McCollough CH. Estimating effective dose for CT using dose-length product compared with using organ doses: consequences of adopting International Commission on Radiological Protection publication 103 or dual-energy scanning. AJR Am J Roentgenol. 2010;194(4):881-9. 
19. Gyftopoulos S, Hasan S, Bencardino J, Mayo J, Nayyar S, Babb J, et al. Diagnostic accuracy of MRI in the measurement of glenoid bone loss. AJR Am J Roentgenol. 2012;199(4):873-8.

20. Huysmans PE, Haen PS, Kidd M, Dhert WJ, Willems JW. The shape of the inferior part of the glenoid: a cadaveric study. $\mathrm{J}$ Shoulder Elbow Surg. 2006;15(6):759-63.

21. Tian CY, Shang Y, Zheng ZZ. Glenoid bone lesions: comparison between 3D VIBE images in MR arthrography and nonarthrographic MSCT. J Magn Reson Imaging. 2012;36(1):231-6.

22. Bland JM, Altman DG. Statistical methods for assessing agreement between two methods of clinical measurement. Lancet. 1986;1(8476):307-10.
23. Koo TK, Li MY. A Guideline of Selecting and Reporting Intraclass Correlation Coefficients for Reliability Research. J Chiropr Med. 2016;15(2):155-63.

24. Biswas D, Bible JE, Bohan M, Simpson AK, Whang PG, Grauer JN. Radiation exposure from musculoskeletal computerized tomographic scans. J Bone Joint Surg Am. 2009;91(8):1882-9.

Publisher's note Springer Nature remains neutral with regard to jurisdictional claims in published maps and institutional affiliations. 